\title{
Growth Cone Configuration and Advance: A Time-Lapse Study Using Video-Enhanced Differential Interference Contrast Microscopy
}

\author{
John M. Aletta a and Lloyd A. Greene ${ }^{a}$ \\ Department of Pharmacology, NYU Medical Center, New York, New York 10016
}

We have analyzed the dynamics of growth cone configuration using video-enhanced contrast differential interference contrast microscopy. Regenerating neurites from NGFtreated PC12 cells and sympathetic neurons were observed in real time during their elongation and reviewed by timelapse video recordings. This technique provided a high-resolution view of motile growth cone elements including filopodia, microspikes, lamellipodia and ruffles. On the basis of our observations, a multistage model for growth cone advance is proposed. Elongation commences with lamellipodial spreading. If the newly extended lamellipodium does not retract or lift off the substrate in the form of a ruffle, a second phase-consolidation-occurs, in which the lamellipodium thickens as it fills with cytoplasm and organelles. The consolidated area then undergoes further transformation into an area of neuritic shaft as new lamellipodia form at the leading and peripheral zones of the distal process. We never observed filopodia or microspikes contracting to propel the growth cone forward. We also noted that elongating tips generally had large varicosities within $20 \mu \mathbf{m}$ of their leading edges. These may play a role in neurite outgrowth and in the formation of smaller, synaptic vesiclecontaining varicosities. The dynamic behavior of the growth cone was under the control of NGF. Withdrawal of NGF resulted in the disappearance of motile structures and cessation of growth, while readdition of NGF triggered the rapid reappearance of these structures and the resumption of growth.

The high-resolution video microscopy of living growth cones provides necessary baseline information, as well as a bioassay paradigm, for future studies on the molecular mechanisms of nerve growth.

Growth cones, the motile endings of growing nerve fibers, wend their way through the complex microenvironment of the developing organism toward their targets by means of largely un-

Received Apr. 22, 1987; revised Sept. 14, 1987; accepted Sept. 14, 1987

We wish to thank Dr. Mary E. Hatten for generously making her high-rcsolution videomicroscopy facilities available to us, Dr. Carol Mason for critical comments on the manuscript, Dr. James Edmondson for many helpful discussions, and Dr. Russell Rydel for generously providing sympathetic neuron cultures. We also thank Federico Negri and Yvel Calderon for their help throughout the preparation of the manuscript.

This work was supported by NIH Grant NS 16036 and by a basic research grant from the March of Dimes Birth Defects Foundation awarded to L.A.G. J.M.A. is supported by NRSA Fellowship NS 07754.

Correspondence should be addressed to John M. Aletta, Department of Pathology, Columbia College of Physicians and Surgeons, Columbia University, 630 W. 168th St., New York, NY 10032.

a Present address: Department of Pathology, Columbia College of Physicians and Surgeons, Columbia University, 630 W. 168th St., New York, NY 10032.

Copyright (C) 1988 Society for Neuroscience $0270-6474 / 88 / 041425-11 \$ 02.00 / 0$ known mechanisms. During elongation of the axon in primary cell culture, new surface membrane is incorporated mainly in the region of the growth cone (Bray, 1970; Pfenninger and Maylie-Pfenninger, 1981). The sequence of events leading from this incorporation to increases in neurite length and to the dynamic behavior of the growth cone have been difficult to discern directly. For example, it is not entirely clear whether the increase in fiber length occurs in conjunction with tension generated on the cone by its filopodia or microspikes (Bray, 1982; Bunge et al., 1983) or with the bulk flow of cytoplasm "pushing" forward from the neuritic shaft (Weiss and Hiscoe, 1948; Lasek, 1981; Bunge et al., 1983). Bray and Chapman (1985) have proposed a direct role for microspike/filopodial movement in the advance of the growth cone. Recently, Goldberg and Burmeister (1986) have argued, on the basis of observations of cultured molluscan neurons made using K. D. Allen's video-enhanced contrast differential interference contrast microscopy (AVEC-DIC), that the rolc of filopodia in the clongation phase of growth is more likely one of structural support than one of active contraction or shortening against an adhesive substrate.

In addition to the mechanism of growth cone advance, another issue of importance is the regulation of growth cone properties and behavior by neurotrophic factors. Although NGF is clearly capable of influencing the direction of growth (Gundersen and Barrett, 1980) and the shape of neurite endings (Seeley and Greene, 1983; Connolly et al., 1987), it has not been possible to observe the dynamics of these events in living cells with high resolution, nor has it been altogether clear how such growth cone actions of NGF are related to neurite elongation. Whether specific features of growth cones are likely to play active or passive permissive roles in neurite elongation can best be answered by analyzing the dynamic aspects of growth cone shape and advance.

We have, therefore, extended the analysis of NGF-dependent growth cone configuration and motility using a high-resolution AVEC-DIC system (Allen et al., 1981). This system offers several advantages for microscopic examination of growth cones. For instance, it allows observation of neuritic growth in living cells in real time and by time-lapse with an optical resolution and contrast just below that of scanning electron microscopy. In addition, by optically sectioning through the growth cone, a plane of focus suitable for viewing intracellular organelles can be obtained. In the present study, we have observed the growth cones of NGF-responsive rat sympathetic neurons and PC12 cells. In the presence of NGF, both cell types grow and maintain long, branched neurites. PC12 cells are of particular interest because they can be easily cultivated in large quantitites for future correlative biochemical studies of the molecular mechanisms of neurite growth (Greene and Tischler, 1976). 
Our present results provide a model for the dynamics by which forward advance of growth cones occurs and further characterize the role of NGF in this process (Seeley and Greene, 1983; Connolly et al., 1985, 1987). Growth was also correlated with the presence of neuritic expansions or "varicosities" 10 $20 \mu \mathrm{m}$ proximal to the leading edge of the growth cone. Some of the data contained in this study have been previously presented in abstract form (Aletta et al., 1986).

\section{Materials and Methods}

Cell culture. Holes, $12 \mathrm{~mm}$ in diameter, were punched in $50 \times 9 \mathrm{~mm}$ plastic petri dishes (Falcon 1006) and \#1 glass coverslips (Fisher) were attached to them with paraffin to create a microwell cell culture system. The glass was then coated with a mixture of poly-L-lysine hydrobromide (Sigma; $5 \mu \mathrm{g} / \mathrm{ml}$ ) and collagen (Bornstein, 1958), 1:75, in 30\% ethanol and dried overnight. Coverslips were rinsed once with sterile deionized water, allowed to dry, and were either used immediately or within $3 \mathrm{~d}$. Preliminary experiments on glass coverslips coated with Matrigel (Collaborative Research) yielded similar results.

PC12 cells were grown on collagen-coated plastic dishes for 1 week or longer in the presence of NGF $(50 \mathrm{ng} / \mathrm{ml}$; Mobley et al., 1976), as described previously (Greene and Tischler, 1976). These cells were triturated from the plastic dishes, replated in RPMI medium (Gibco) containing $10 \%$ donor horse serum, $5 \%$ fetal calf serum (KC Biological) and $50 \mathrm{ng} / \mathrm{ml} \mathrm{NGF}$ on the glass coverslips described above, and allowed to regenerate their neurites for $12 \mathrm{hr}$ to $4 \mathrm{~d}$ in a $7.5 \% \mathrm{CO}_{2}$ incubator at $35^{\circ} \mathrm{C}$ before microscopic examination.

The source of rat sympathetic neuron growth cones was either explants of superior cervical ganglia (postnatal day 2 or 3) or dissociated cell cultures of several postnatal day 3 superior cervical ganglia. All were cultured under the same conditions used for PC12 cells, except that some cultures were treated for $1-3 \mathrm{~d}$ with $10 \mu \mathrm{M}$ cytosine arabinoside to reduce the non-neuronal cell population. Observations using AVECDIC microscopy were made $2-10 \mathrm{~d}$ after plating.

Video microscopy. Immediately prior to the microscopic examination, another \#1 glass coverslip was placed over the microwell culture, which was then sealed with silicone grease. The microscope stage was maintained at $36 \pm 1{ }^{\circ} \mathrm{C}$ with an air curtain (Ycllow Springs Instruments Model 72) and, judging from the pH dye indicator in the RPMI medium, $\mathrm{pH}$ control was also maintained in the sealed well for at least $12 \mathrm{hr}$.

We used an inverted light microscope (Zeiss IM) equipped with Nomarski differential interference contrast optics, a $50 \mathrm{~W}$ mercury arc lamp, infrared filter, $63 \times(1.4$ N.A.) oil-immersion Planapochromat objective, and a 1.4 N.A. oil-immersion condenser. The microscope image was projected to a Hamamatsu Model C1965-01 video camera containing an analog circuit for contrast enhancement. The image was displayed on a Sony television monitor (PVN-122; 1000 lines of horizontal resolution) for real-time observation. Video images were recorded either on a laser-operated, monochrome, high-resolution optical disc recorder (Panasonic TQ-2025F; 450 lines of horizontal resolution) or by an NEC U-matic video cassette recorder (VC9507) connected to the Sony monitor. When using the optical disc recorder, images were generally recorded once every $4 \mathrm{sec}$. With the videotape recorder, continuous video records were made at time compressions of either $1: 9$ or 1:18. Further details pertaining to this particular imaging systern can be found in a recent publication (Edmondson and Hatten, 1987). Access to this equipment was generously provided by Dr. Mary E. Hatten, Department of Pharmacology, NYU School of Medicine. The photographs presented in this report were taken directly from the monitor screen on $35 \mathrm{~mm}$ technical pan film (Kodak; Estar-AH Base 2415). The direction of optical shear in all the micrographs is perpendicular to the bottom edges of the figures.

Recording protocols. During our experiments, we came to the provisional conclusion that uninterrupted exposure of growth cones to the light source for longer than $30-40 \mathrm{~min}$, even with heat-reducing filters, could be deleterious to these structures. After this time, many growth cones became much less motile and only sometimes regained their activity after several hours without illumination. Given these circumstances, growth cones were, therefore, observed under either one of 2 protocols. Short-term observations of motility and shape changes were generally for $30 \mathrm{~min}$ or less. Each experimental field was observed in real time, as well as recorded in time-lapse for later analysis. Most experiments were recorded on optical discs at 1 frame $/ 4 \mathrm{sec}$. A distinct advantage of this system is that it permits us to view the frames of any sequence either individually, in quick succession, or played back at speeds equivalent to, less than, or greater than the real time in which the action was recorded. Long-term observations over the course of several hours were also carried out to study the extensive shape changes that occur during growth. In these cases, the cones were exposed to the light source at intervals of $20 \mathrm{~min}$ or more for only the short period needed to record either single images (approximately $1 \mathrm{~min}$ ) or examples of interesting motile behavior (less than $5 \mathrm{~min}$ ).

Withdrawal and readdition of NGF. NGF was removed from PC12 cell cultures by a series of washes over several hours (Seeley and Greene, 1983). In order to reintroduce NGF to growth cones during continuous observation in the sealed chamber, culture dishes were modified slightly by constructing a small grooved aperture on the edge of the microwell into which was glued (Devcon Epoxy) a short segment of $0.25 \mathrm{~mm}$ i.d. polyethylene tubing. By attaching a $10 \mu l$ syringe (Hamilton) filled with prewarmed, $\mathrm{pH}$-adjusted NGF $(2.5 \mu \mathrm{g} / \mathrm{ml})$ in RPMI culture medium plus sera, it was possible to reliably deliver $5 \mu \mathrm{l}$ of the NGF solution to the culture (containing a total volume of approximately $200 \mu \mathrm{l}$ ). The precise final concentration of NGF was not clear, owing to inefficient mixing. On the basis of several trials with dye injections, which showed that the low-molecular-weight solute remained localized mainly around the site of delivery for at least $15 \mathrm{~min}$, we studied only growth cones within $2-4 \mathrm{~mm}$ of the tubing inlet.

\section{Results}

Growth cones of NGF-treated PC12 cells and sympathetic neurons exhibit a remarkable variety of shapes and activities, ranging from simple, quiescent club-shaped endings to rather ornate, highly motile endings that either change configuration without forward locomotion or that actively advance. Retraction, too, sometimes occurs ( $n=8$ of 113), although these cases are not considered here. Because of this variety, we have analyzed the behavior of a relatively large number of growth cones (PC12 cells, $n=95$; sympathetic neurons, $n=18$ ). Keeping in mind this variability, we describe below the most distinctive features of the neurite endings observed. Generally, there was little difference between the form and behavior of PC12 cells and of sympathetic neuron growth cones, and the descriptions given here refer to both unless specifically noted.

\section{Elements of the neurite ending}

Growth cone. As viewed by AVEC-DIC, the growth cone proper (see Fig. 1) consists of 2 operationally defined areas. The proximal portion is spread several microns in diameter and is well attached to the substrate, but can be displaced during elongation. This area contains intracellular organelles and is relatively nonmotile; in contrast, the more distal reach of the cone is much thinner, more motile, and organelle-poor (see Figs. 1, 2). Highly motile lamellipodia, microspikes, and ruffles (described more fully below) are the most prominent features of this more distal area. However, both regions of the growth cone can display microspikes and longer, highly motile filopodia.

The cone is capable of remodeling and assuming a variety of shapes (Figs. 2, 3). Such changes can occur within intervals of tens of minutes. For example, cones displaying a predominantly lamellipodial form can evolve into cones with many more filopodia and microspikes (Fig. 2) and vice versa. Other structures are interchangeable. Ruffles can transform into lamellipodia (Fig. 4) and varicosities can migrate forward and merge with the cone (not shown), or the cone may form and leave behind a varicosity as elongation proceeds (Fig. 3).

Filopodia/microspikes. Motile, thread-like projections fell into 2 extreme categories of length. We have arbitrarily designated projections of less than $10 \mu \mathrm{m}$ in length as microspikes, and those longer than $10 \mu \mathrm{m}$ as filopodia (cf. Fig. 2). On this basis, 


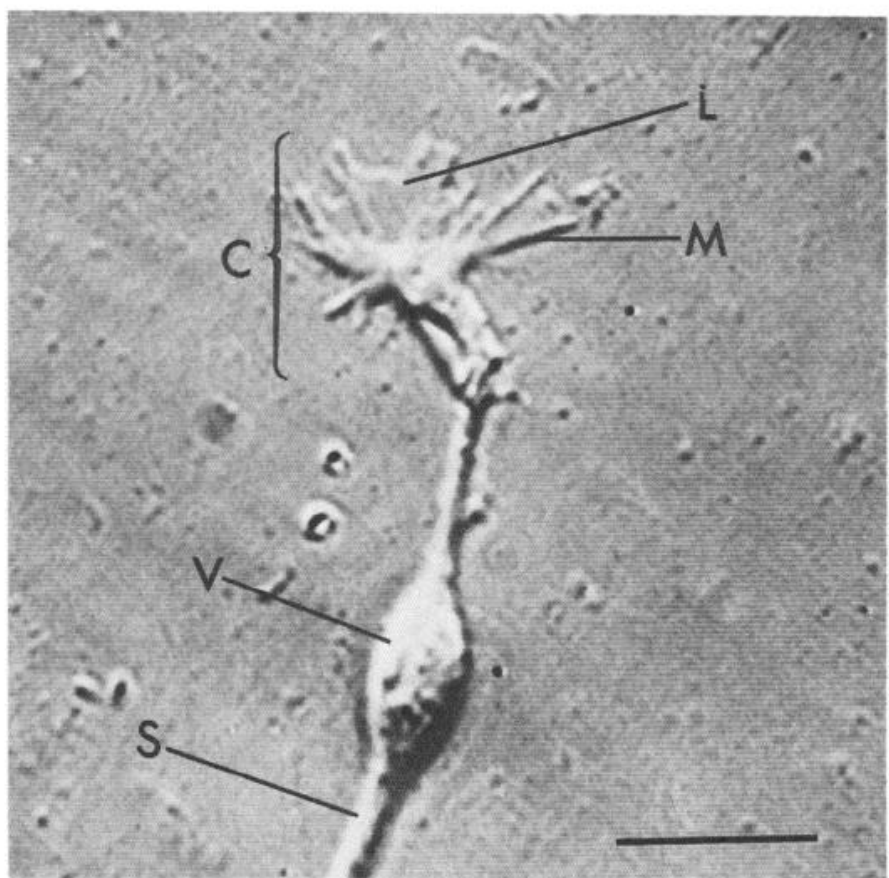

Figure 1. Growth cone elements. The actively elongating PC12 cell neurite shown here consists of a neuritic shaft $(S)$ leading into a neuritic varicosity $(V)$ and the growth cone proper $(C)$. Extending from the latter are lamellipodia $(L)$, and microspikes $(M)$. Calibration bar, $8 \mu \mathrm{m}$.

the majority of projections are microspikes, most of which are less than $7.5 \mu \mathrm{m}$ long $(92 \% ; n=347)$. As previously observed (cf. Nakai and Kawasaki, 1959), motile microspikes are formed and resorbed in large numbers at the growth cone, particularly at the lateral margins and leading edge of the distal cone region, and relatively infrequently at neuritic shafts and varicosities. The average lifetime of 10 randomly chosen microspikes $( \pm$ SD) was $3.2 \pm 1.5 \mathrm{~min}$, with a range from 0.5 to $5 \mathrm{~min}$. Like filopodia, microspikes can sometimes extend off the substrate.

Filopodia generally range from 10 to $25 \mu \mathrm{m}$ in length (the longest observed here was $34 \mu \mathrm{m}$ ) and can be seen in real time to extend or retract within seconds. The average length $( \pm \mathrm{SD})$ of 41 filopodia was $16 \pm 7 \mu \mathrm{m}$. These projections do not appear to be particularly well attached to the substrate, and usually move in and out of the plane of focus, probing the environment in all 3 dimensions (cf. Figs. 2, 5). In general, filopodia are longer lived than microspikes $(18.9 \pm 12.2 \mathrm{~min}$, with a range from 3.5 to $38 \mathrm{~min} ; n=8$ ). A growth cone may have up to 10 filopodia or none at all, and this number is not static, often changing over the course of seconds to minutes because of the interchangeability of microspikes and filopodia.

We saw no evidence that filopodia or microspikes exert tension on the growth cone, thereby resulting in forward locomotion. However, in several instances, filopodia were observed to attach to, and pull on, neuritic shafts, but the direction of subsequent growth did not necessarily follow the filopodial attachment (data not shown).

Lamellipodia. Another prominent feature clearly delineated by AVEC-DIC is the lamellipodia (Abercrombie et al., 1970; Porter and Fitzsimons, 1973), which appear as excrescences of very thin sheets of membrane and cytoplasm at the growth cone's leading edges (cf. Figs. 1, 2, 5). Lamellipodia form rapidly, over a time course of, at most, tens of seconds, by the forward flow of the cone's membrane, which results in a gentle gliding across the substrate (Fig. 5). This lateral gliding motion suggests that the substrate attachments formed by the lamellipodia are
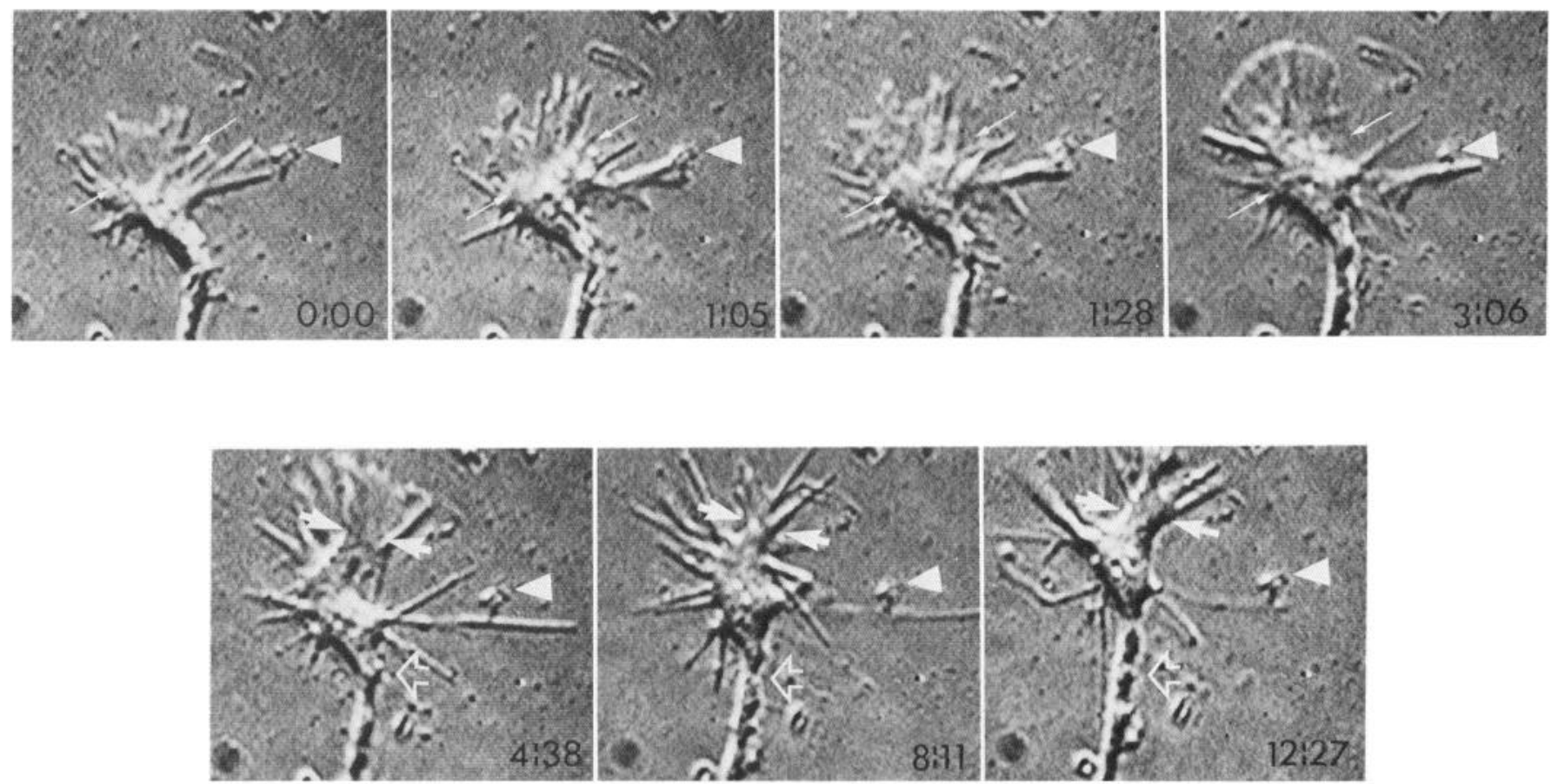

Figure 2. Lamellipodial filling by influx of cytoplasm and organelles. The relative time of recording (in min) of the same growth cone is denoted in the lower-right corner of each panel. A cluster of debris marked by the large white triangle serves as an invariant reference point. The upper sequence shows the filling of an area denoted by the 2 small arrows. In the lower sequence, filling occurs between the 2 large filled arrowheads. An area of the proximal cone in the process of undergoing conversion to neurite ("stabilization") is indicated by the unfilled arrowhead. 

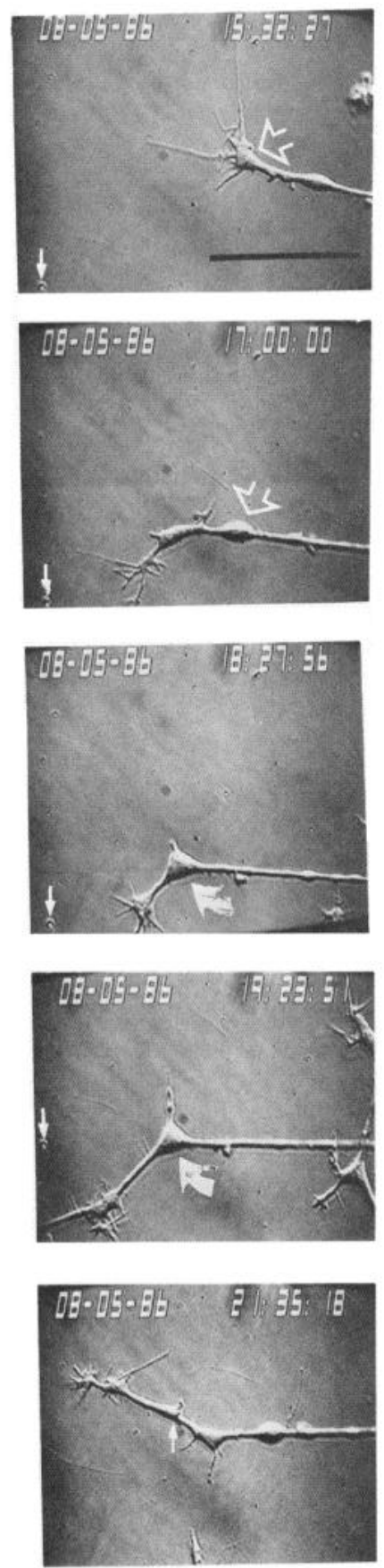
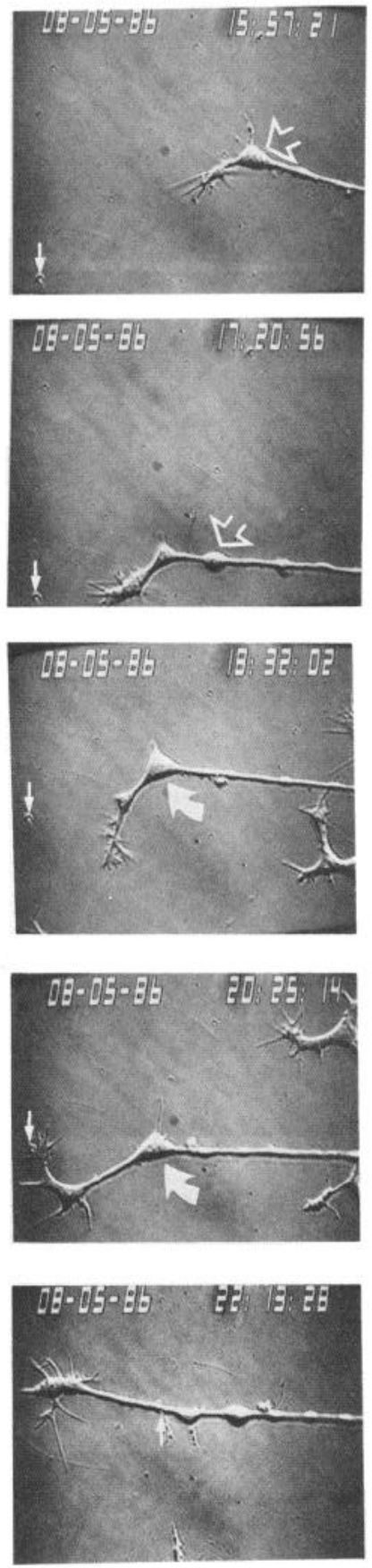
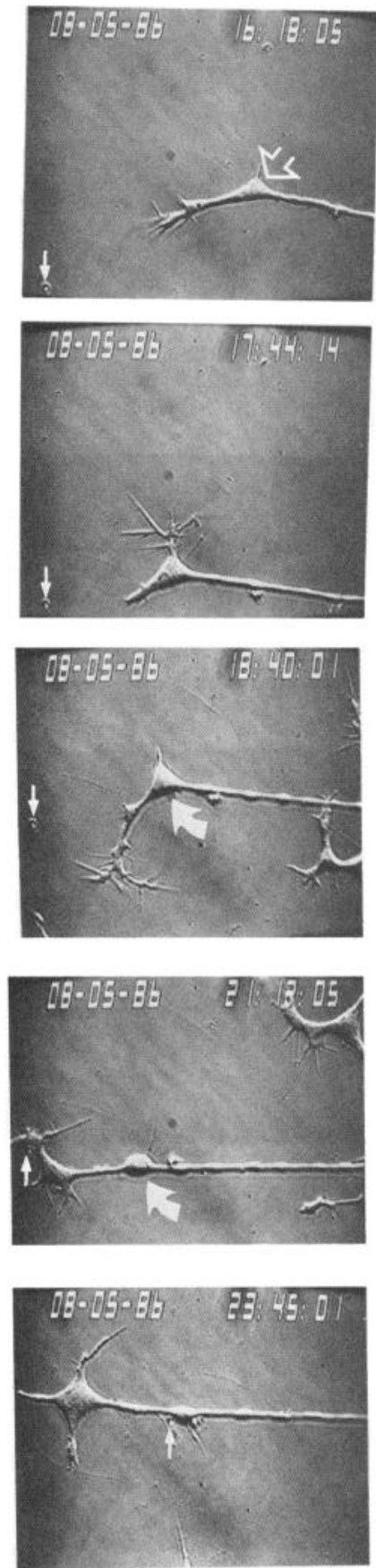
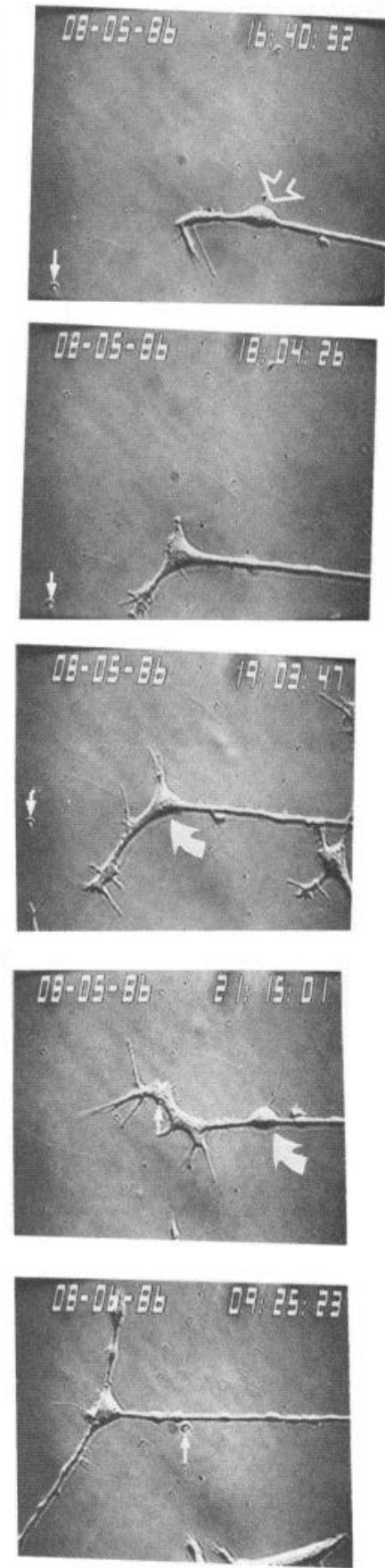

Figure 3. Transfigurations of PC12 cell neuritic dilations (varicosities) and growth cones during long-term observation. Two instances in which cones transform into varicosities are indicated by the arrowheads and curved arrows. The lower 4 frames document the gradual metamorphosis of 2 large varicosities into smaller swellings. An invariant reference point in the fields of views is marked on the photographs (arrow). During the first $6.5 \mathrm{hr}$ of observation, an elongation of $67 \mu \mathrm{m}$ occurred (mean rate, $10 \mu \mathrm{m} / \mathrm{hr}$ ). Periods of quiescence were interposed with others of extension more rapid than the overall average (maximal rate, $39 \mu \mathrm{m} / \mathrm{hr}$ ). The growth cone was observed periodically for short times, as described in Materials and Methods. The intervals between successive frames in this and subsequent figures can be deduced from the actual clock times at the upper righthand corners. Bar, $20 \mu \mathrm{m}$.

transient. Lamellipodia do not appear to contain visible, particulate organelles, and it is likely that exceedingly little cytoplasm is present in such thin structures. Often, lamellipodia appear to form between microspikes (Figs. 1, 2, 5); sometimes they form between the bases of adjacent filopodia or, rarely, at the ends of single filopodia. In most cases, lamellipodia are withdrawn or resorbed back into the growth cone. During the active gliding phases of a lamellipodium's formation, all or part of its leading edge can frequently detach from the substrate and form a ruffle (Fig. 4) or what others have called "unattached veils" (Tosney and Wessells, 1983).

Ruffles. AVEC-DIC was particularly effective for visualizing the dynamics of growth cone ruffles (see also Abercrombie and Ambrose, 1958; Bray and Bunge, 1973; Connolly et al., 1985, 1987). From our observations, ruffles can be classed into 2 groups. The more typical (type I) is that seen at the leading edge 

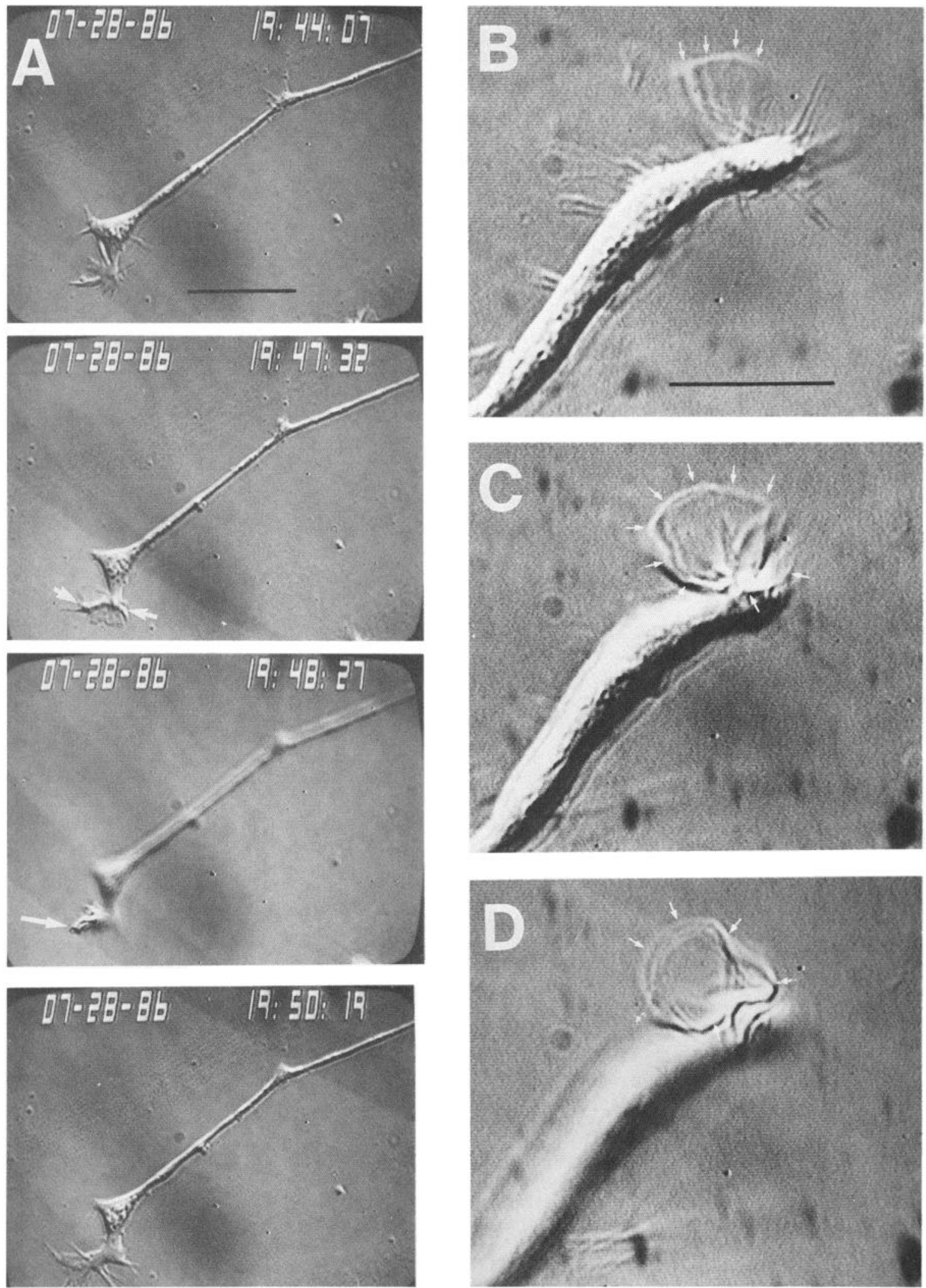

Figure 4. Ruffles. A, Leading-edge ruffle. This sequence demonstrates the formation and lamellipodial fate of a PC12 cell ruffle at the growth cone's leading edge over the course of $61 / 4 \mathrm{~min}$. Arrowheads mark the margins of the nascent ruffle at 19:47:32, and the arrow in the next panel points out a portion of the ruffle in an optical plane well above the substrate level of the dish. The bottom panel shows the transformation of the ruffle into an enlarged lamellipodium with numerous microspikes. Bar, $15 \mu \mathrm{m}$. $B-D$, Funnel-shaped ruffle. Rarely observed structures, these PC12 cell surface ruffles (arrows) were highly mobile but were never associated with forward locomotion. $B$, Made with the plane of focus at the neurite's exposed surface; $C$, with focus above the plane of the neurite through the midsection of the ruffle; $D$, well above the neurite at the top of the ruffle. All 3 frames were taken within less than 1 min of time. Bar, $10 \mu \mathrm{m}$. 

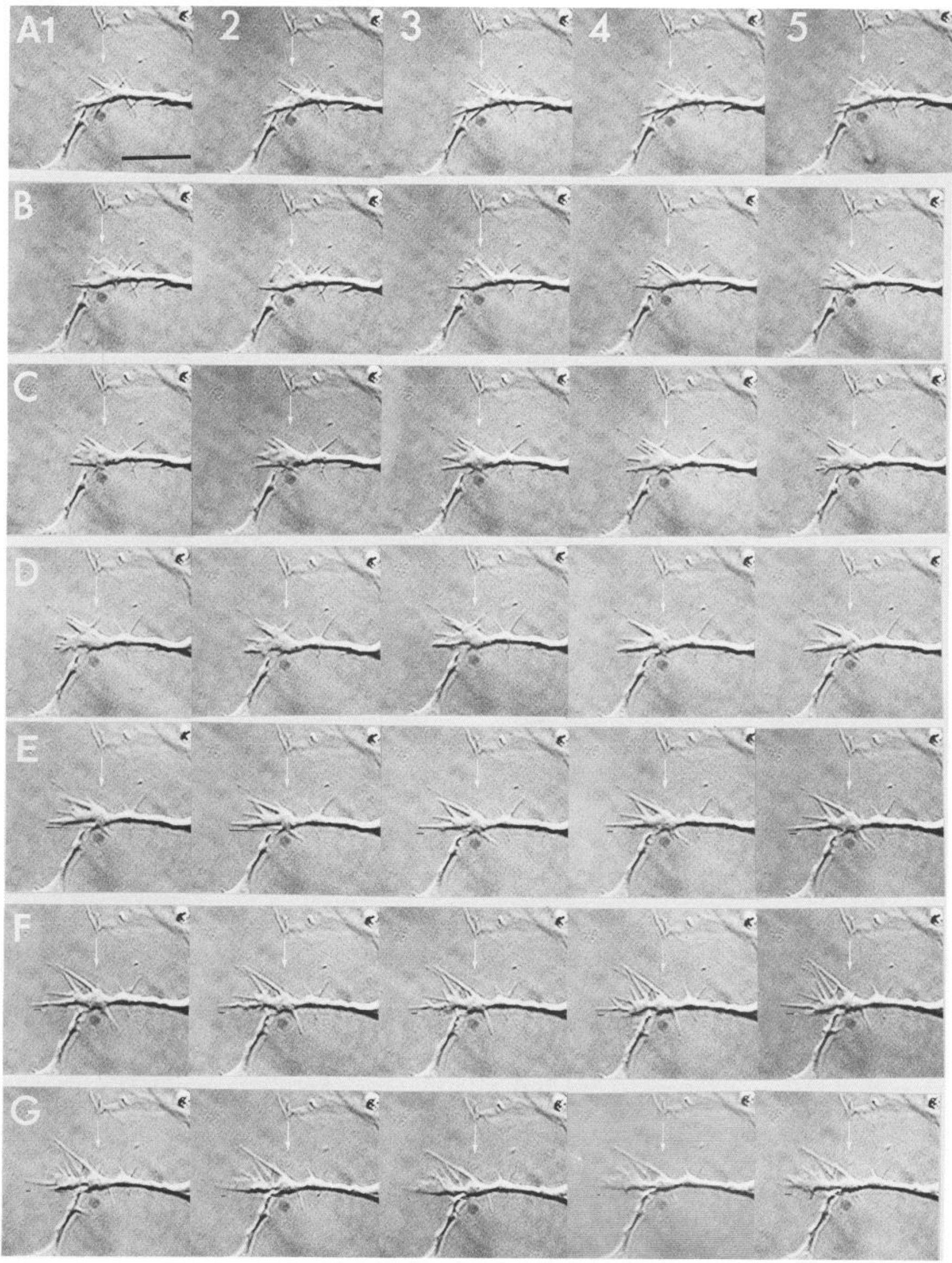
of, or just behind, lamellipodia (Fig. 4A). These form when portions of lamellipodia lift off the substrate (Ingram, 1969). These structures can extend vertically for considerable distances and can either reattach to the substrate to reform lamellipodia, be slowly resorbed into the cone, or fold backwards lengthwise to collapse rapidly back into the cone. Such changes can occur within $30 \mathrm{sec}$ to several minutes. This again exemplifies the interchangeability among growth cone elements.

The second type of ruffle (type II) was much more rarely observed. These are much larger than the first group and are sometimes wider in diameter than both the neuritic shaft and the growth cone itself. They appeared to be funnel-shaped (Fig. $4, B-D$ ) or sometimes had tightly wrapped membranes in a flowerlike configuration. Because the latter structures were so large, it was difficult to observe them in a single optical plane (see Fig. 4), and conseguently they were best observed while focusing through them in real time. Both forms of type II ruffle originated on the surface or leading edge of growth cones and were relatively long-lived (20-30 min). Type I ruffles were often seen during $\mathrm{PCl} 2$ cell and sympathetic growth cone advance, but type II ruffles were seen only on PC12 cell growth cones.

\section{Growth and consolidation}

Observations of many growth concs, cither in real time or by examination of video time-lapse recordings at a variety of playback speeds, have led us to conclude that, under the conditions studied, NGF-promoted neurite extension always commences with the formation of lamellipodia at the leading and peripheral edges of the growth cone (Figs. 2, 5). If these structures are not resorbed or do not lift off the substrate to form ruffles, they appear to thicken, apparently through the flow of cytoplasm from more proximal portions of the cone and neurite (Figs. 2; 5 , row $\mathrm{C}$ ). The entry of visible organelles has been the most reliable sign that resorption or ruffling is unlikely and that stable advancement of the growth cone will occur. When this "filling" takes place, the lamellipodia become less motile and become part of the proximal, well-attached area of the cone. The transformation of motile, transitory lamellipodia into a thickened, well-attached cone is termed by us "consolidation," and represents the means by which neurite growth occurs. As consolidation proceeds, the distal portions of the cone continue their motile and exploratory behavior and form new lamellipodia (Fig. 5, row G). Each increment of consolidation adds $2-5 \mu \mathrm{m}$ to the length of the neurite. Lamellipodial additions can occur in rapid succession, producing elongation at rates of up to 70 $\mu \mathrm{m} / \mathrm{hr}$. The average rate $( \pm \mathrm{SEM})$, obtained from 50 advancing PC1 2 cell growth cones (extrapolated from observation periods of 17-30 min) was $20 \pm 2 \mu \mathrm{m} / \mathrm{hr}$. For sympathetic neurons, the value was quite similar: $18 \pm 4 \mu \mathrm{m} / \mathrm{hr}(n=11)$. As has previously been documented (Argiro et al., 1984), neurite growth was saltatory, in that periods of rapid advancement were interspersed with periods of relatively little or no elongation.

While numerous microspikes were often present at the edges of advancing growth cones, neither these structures nor filopodia were ever observed to produce elongation via the generation of tension. The numbers, distributions, and length changes of microspikes and filopodia on the growth cone were quite variable, precluding a discernible mechanism involving extension via a concerted shortening of longitudinally oriented filamentous processes. Lamellipodial outflow and consolidation occurred, in many instances, on areas of the cone with few microspikes. In other cases, microspikes formed at the cone's edge just prior to the formation of lamellipodia between them (Fig. 5, row A), and on still other occasions, microspike-like outlines became apparent within lamellipodia only after these thin sheets of membrane were formed (see, for instance, Fig. 5, rows B and C). In any case, the neurite did not appear to be pulled forward by microspikes and/or filopodia, but, rather, advanced by the accrual of new material via consolidation of lamellipodia.

While lamellipodial consolidation and advance of the neurite take place toward the distal portion of the growth cone, a transformation also occurs at the more proximal cone area. Ultimately, this region, which is still somewhat motile, plastic in shape, and well spread, changes form and comes to be part of the nonmotile, cylindrical neuritic shaft (Figs. 2, 5). We have termed this cone-to-shaft transition "stabilization," since it represents the last discernible step in neurite elongation.

The growth events that occur at the distal and proximal areas of the cone must involve changes in adhesion to the substrate. Attachment of lamellipodia is transient, i.e., on the order of seconds, unless consolidation is accomplished. The consolidated areas of the cone, by contrast, appear to adhere well to the substrate. On the other hand, the neuritic shaft, into which the proximal cone transforms, appears to be the least well-attached portion of the cell. One conscquence that reflects these differences is that, whereas the growth cone may follow a meandering path as it advances, the neurite shaft, being poorly adherent, follows a generally straight line between the cone and attachment sites closer to the cell soma (Fig. 3).

\section{Varicosities and neurite elongation}

As observed by AVEC-DIC (c.. Figs. 1, 3, 6), 1-3 swellings or "varicosities" are typically present along the shaft of the neurite just proximal to the growth cone proper (average distance \pm $\mathrm{SEM}=13 \pm 5 \mu \mathrm{m}$ behind the leading edge; $n=82$ ). Other dilated regions, along the more proximal neuritic shaft at irregular intervals and in varying quantities, were of considerably smaller caliber (Fig. 3, last panel). The most characteristic dilation encountered near the growth cone is an ellipsoidal structure (Fig. 1). This form, however, is not static, and besides incrcasing or decrcasing its axial dimensions, it can undergo gradual transfigurations (Figs. 3,6) into a variety of other shapes (i.e., triangles, diamonds, or spindles). Such varicosities can translocate at several microns per hour along the shaft of the neurite in either direction. In addition, intracellular organelles (see below) were frequently observed within varicosities. Although such particles can exit these areas and move toward either the growth cone or the cell body, one or more large clusters

\section{$\leftarrow$}

Figure 5. Growth and consolidation. This 3 min time-lapse sequence of a growing PC12 cell neurite was taken from an optical disc recording. One frame was recorded every $4 \mathrm{sec}$. $A-E$, Each successive frame, at 4 sec intervals, from 1 min, 40 sec of the sequence. $F$, $G$, Every other frame from the next $1 \mathrm{~min}, 20 \mathrm{sec}$ (at approximately $8 \mathrm{sec}$ intervals). Progressive lamellipodial spreading between microspikes is shown in $A$ and $B$. Thickening and consolidation of the newly extended lamellipodium can be seen from $B, 5$ through $C, 5$. Consolidation continues, and by frame

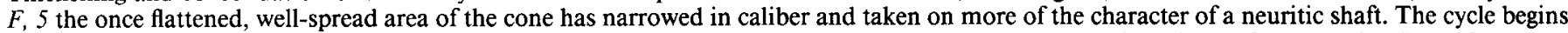
again in $G$ with another round of lamellipodial spreading at the tip of the neurite. The arrow marks an invariant reference point. Bar, $10 \mu \mathrm{m}$. 

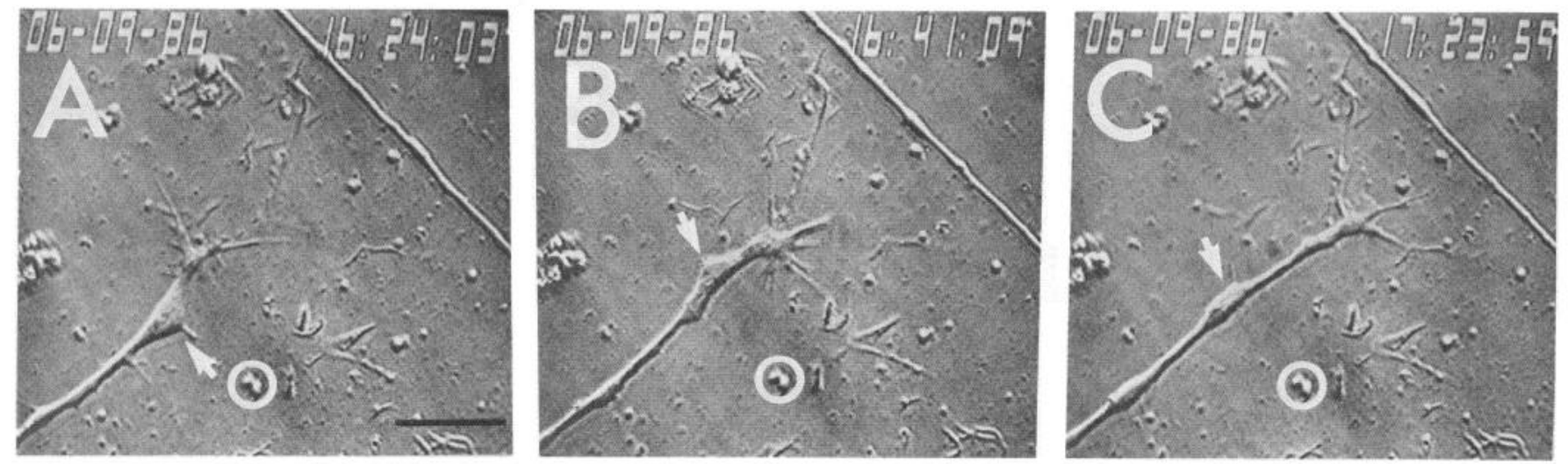

Figure 6. Remodeling of a sympathetic neuron growth cone-associated varicosity. The varicosity (arrowhead) fuses with its growth cone (B), after which it maintains its position while the growth cone continues its forward migration $(C)$. An invariant landmark is circled in $A-C$. Bar, $10 \mu$ m.

of organelles were always present in the varicosity during elongation.

The varicosity appears to be intimately associated with the advancing growth cone. As noted above, this structure can migrate along with and behind the moving cone (data not shown). Alternatively, as consolidation occurs, the more proximal region of the cone can become separated from the advancing distal cone and take on the characteristics of a varicosity. Thus, at least some of the varicosities arise directly from consolidated portions of growth cones. Figure 3 contains a sequence that illustrates several varicosities forming from the advancing cone over a time course of several hours.

Our observations also suggest that varicosities present in association with growth cones could play a role in neuritic growth. While elongation is occurring, material can be seen, in real time and in time-lapse, moving from the varicosity, which often changes in size and shape during the advance. Often, the varicosities get much thinner, as if they were supplying material for the advancing edge and consolidating cone. On some occasions, these structures even diminish completely, so that only shaft remains where a varicosity had been located (data not shown). When this occurs, motility and advancement of the cone typically cease until a new varicosity forms.

To test the association between neurite elongation and the presence of a varicosity, these 2 characteristics were scored for 67 PC12 cell neurites (Table 1). A varicosity was present within $20 \mu \mathrm{m}$ of over $90 \%$ of the cones that advanced. In contrast, growth did not occur in more than $60 \%(7 / 11)$ of the cases in which varicosities were absent. Evaluation of these data by the chi-squared exact test for $2 \times 2$ tables revealed that this difference is significant at $p<0.01$.

Table 1. Chi-squared exact test for $2 \times 2$ tables $^{a}$

\begin{tabular}{lll} 
& $(+)$ Varicosity & $(-)$ Varicosity \\
\hline (+) Growth & 46 & 4 \\
(-) Growth & 10 & 7
\end{tabular}

All video recordings of growth cones of $\mathrm{PC} 12$ cells that spanned $30 \mathrm{~min}$ from the onset of each observation were submitted to this analysis. Neurites that elongated $4 \mu \mathrm{m}$ or more during this period are included in the (+) Growth bins. Those neurites found in the (-) Growth bins did not elongate significantly (less than or equal to $2 \mu \mathrm{m}$ ) during the observation period. Neurites that possessed at least one varicosity, as described in the text, are included in the $(+)$ Varicosity bins, those without, in the $(-)$ Vericosity bins. $n=67 ; d f=1 ; p<0.01$.

${ }^{a}$ Bailey (1959).
A smaller number (18) of sympathetic neuron growth cones was also reviewed for the presence of varicosities. Dilated regions, as described above and within $20 \mu \mathrm{m}$ of the growth cone, were observed in 10 cases (see, for example, Fig. 6). Of these, 9 advanced $3 \mu \mathrm{m}$ or more within $30 \mathrm{~min}$ of observation. Of the remaining 8 without clear growth cone-associated varicosities during the recording period, only 4 underwent similar growth. The average lifetime of varicosities associated with sympathetic neuron growth cones appeared to be less than that in the case of PC12 cells. This was particularly so for the neurons within the first day or two after plating. As a consequence of this transient nature, a much smaller proportion of neurons in the early cultures was observed to possess varicosities at any given moment.

\section{Response to NGF withdrawal and readdition}

To further examine the dynamic aspects of NGF's regulatory action on growth cone structure and function at the high resolution afforded by AVEC-DIC, growth cones were examined in real time and in time-lapse recordings after NGF deprivation for $7 \mathrm{hr}$ or more, and then upon and for $20 \mathrm{~min}$ or more after NGF readdition. In the NGF-deprived cultures, nearly all the neurites examined were blunt-ended (Fig. 7, $A, B$ ) and, except for a few filopodia and microspikes, were not motile and never locomotive. When NGF was reintroduced into the culture medium in the vicinity of the growth cones, motility was observed within $45 \mathrm{sec}$ to $3 \mathrm{~min}$. This included the formation of microspikes, lamellipodia, and ruffles at the process tip (Fig. 7; see also Fig. 4, which comes from a sequence 5-11 min after readdition of NGF). Elongation, as characterized above, also commenced within several minutes of NGF readdition. As previously indicated by either phase- or scanning-electron microscopy (Seeley and Greene, 1983; Connolly et al., 1985, 1987), microspike activity transiently appeared along the neurite shaft after NGF was readded (Fig. 7, $D-F$ ). Finally, the movement of intracellular particles within the neurite shaft did not appear to be affected by either NGF withdrawal or readdition.

\section{Discussion}

The aim of the present study has been to observe the dynamic features of growth cones in living cells under high resolution in order to better understand the sequence of events that leads to neurite elongation. Though many of the individual elements seen here have been described before (cf. Nakai and Kawasaki, 

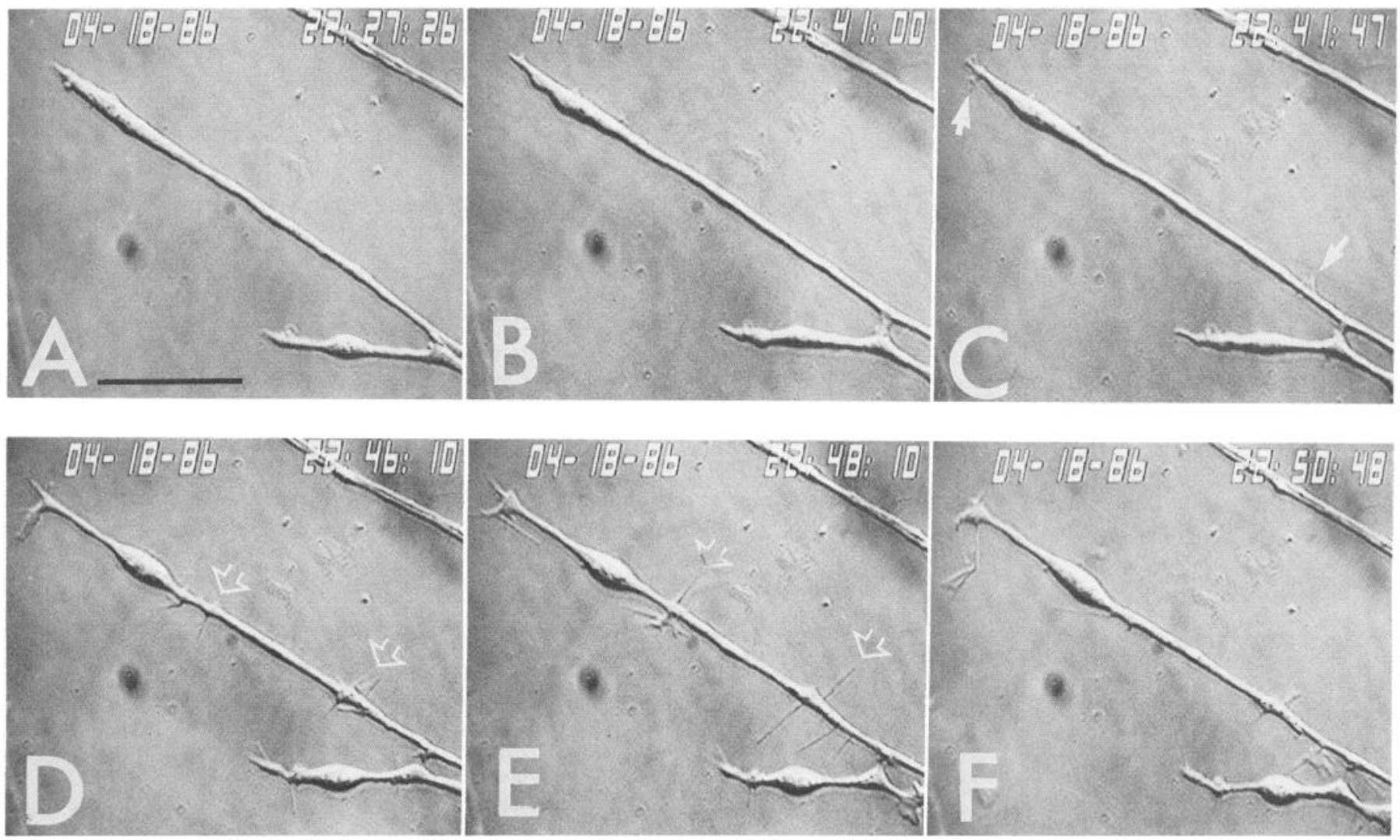

Figure 7. Motile features of elongating PC12 cell neurites are dependent on NGF. NGF was withdrawn from these PC12 cell neurites for 10 hr. None of the highly motile features of typical NGF-treated growth cones were evident $(A)$. NGF was rapidly readded at 22:41:00 (B, and within 1 min motile elements began to reappear $(C$, arrowheads). Microspikes appeared along the neurite shaft (arrowheads in $D, E)$, but were present transiently $(F)$. Bar, $15 \mu \mathrm{m}$.

1959; Johnston and Wessells, 1980), the use of high-resolution AVEC-DIC observations and time-lapse recordings has permitted a dynamic, integrated account of their roles in process outgrowth. In particular, these findings support a protrusive model for elongation and call attention to the interaction of large varicosities with advancing growth cones. They also show, at high resolution, that the dynamic features, and thereby advancement, of the cone may be rapidly controlled by a neurotrophic agent.

\section{Growth cone shape}

The overall morphology of individual growth cones was remarkably variable. This was true both for different growth cones as well as for the same growth cone at different times. Characteristic growth cone shapes have been demonstrated to be an intrinsic property of certain identified adult invertebrate neurons in culture (Haydon et al., 1985). Our results suggest that at least some vertebrate postnatal neurons may possess a less uniform growth cone phenotype. This is in consonance with in vivo studies of growth cone shape along pathways to synaptic targets (Tosney and Landmesser, 1985; Bovolenta and Mason, 1987).

\section{Varicosities}

In addition to lamellipodia, ruffles, and microspikes (cf. Johnston and Wessells, 1980), one or more neuritic expansions, or "varicosities," were also consistently associated with forward locomotion (Table 1). These structures appear similar to the varicosities along axons regenerating from goldfish retinal ex- plants (Koenig et al., 1985) and to the focal swellings of postnatal rodent cerebellar axons (Gravel et al., 1986). Both in the present study and in that of Koenig et al. (1985), varicosities were capable of bidirectional migration along the neurite. Using AVECDIC, we also directly observed movements of organelles from the varicosity into the growth cone. On the basis of this observation and of the correlation between elongation and the presence of a varicosity within $20 \mu \mathrm{m}$ of the advancing growth cone, we propose that these structures may serve as reservoirs of material necessary for neurite growth. The large clusters of organelles seen in growth cone-associated varicosities may be analogous to the large irregular refractile body (LIRB) described by Goldberg and Burmeister (1986) in cultured Aplysia neurons.

Varicosities similar to the type described here may also be involved in functions other than elongation. For instance, retrieval of material from the distal neurite to a nascent varicosity during neurite retraction has been described (Koenig et al., 1985). Disruption of cytoskeletal elements with microtubule-destabilizing drugs also produces swollen regions along the neuritic shaft (Horie et al., 1983; Jacobs and Stevens, 1986), which may signify that neurite retraction at least partially recapitulates the events of growth in reverse. An additional possible role for growth cone-associated varicosities is to develop into the vesicle-containing regions we have observed by transmission electron microscopy along the shaft of the neurite (Greene and Tischler, 1976; J. M. Aletta and K. R. Brown, unpublished observations). These varicosities, located much more proximally along neuritic shafts, resemble the presynaptic elements of en passant synapses formed by the autonomic nervous system 
and appear to be considerably smaller than the varicosities at growth cones (cf. Fig. 3, bottom right photo).

\section{Neurite elongation}

On the basis of observations made with AVEC-DIC, we propose a multistage model of neuritic outgrowth that may be summarized as follows: (1) membrane addition in the form of motile lamellipodia and/or ruffles, (2) consolidation of the newly added area of membrane by inflow of axoplasm and organelles and loss of motility, (3) progressive modification of substrate attachment by various areas of the growth cone (see below), and (4) transition of consolidated regions into neuritic shaft (i.e., stabilization).

Tension generated by shortening or isometric contraction of the microspikes or filopodia against a tightly held substrate has been suggested as a principal means by which axonal elongation is generated (Bray, 1973, 1982; Bray and Chapman, 1985). The role of these structures in neurite elongation, however, has recently been called into question. Marsh and Letourneau (1984) demonstrated the growth of neurites lacking filopodia and lamellipodia and concluded that tension generated by these elements is not required for neurite elongation. Bentley and Toroian-Raymond (1986) also found neurite elongation under conditions in which filopodial formation was impaired. Another recent study in which molluscan growth cones were observed by AVEC-DIC indicated that filopodia facilitate elongation not by the generation of tension, but by guiding the formation of "veils" (lamellipodia) (Goldberg and Burmeister, 1986).

On the basis of our observations, we conclude that filopodial contraction and generation of tension are not the means by which NGF-responsive growth cones advance in our cultures. We never observed microspike shortening (isotonic contraction), which could be construed as pulling the growth cone forward. Lamellipodia, on the other hand, consistently surged forward along and beyond microspikes. Although we cannot categorically rule out the exertion of isometric tension, the numerous instances of ruffling, in the absence of adherent microspikes, with the consequent reattachment of the ruffle and its transformation into a motile lamellipodium (Fig. 4) suggest that such tension is not essential for membrane addition. Thus, our findings are consistent with the view (cf. Marsh and Letourneau, 1984; Caudy and Bentley, 1986; Goldberg and Burmeister, 1986) that microspikes and filopodia are involved in the guidance, rather than in the elongation of neurites. Instead of being pulled forward in toto, the growth cone advances via a persistent cytoplasmic flow into a newly extended lamellipodial appendage.

Despite our negative observations regarding the role of filopodial tension in neurite elongation, our findings cannot necessarily be taken to conclude that such a mechanism does not operate under other conditions or in other species. For instance, Hammarback and Letourneau (1986) have provided evidence that filopodial contact with an area of high adhesivity can serve as a "guidepost" enabling cultured chick dorsal root ganglionic neurons to extend across an intervening substrate of low adhesivity.

\section{Substrate interaction}

Changes in the interaction of growth cone elements with the substrate may play an important role in the advancement process. Our observations, in agreement with prior work (cf. Letourneau, 1979), indicate that the principal attachment site of the neurite ending is beneath the central portion of the growth cone. Elongation commences with lamellipodial spreading and attachment to the substrate. This is transient, being on the order of seconds. In many cases, lamellipodia are withdrawn or lift off the substrate to form ruffles. Only when consolidation occurs do they bccomc part of the relatively stable, well-attached portion of the growth cone. The consolidation step itself may be, in part, influenced by interaction with the substrate. That is, on a poorly adhesive substrate, lamellipodia may be apt to withdraw or ruffle before consolidation can occur, while, conversely, on a strongly adherent substrate, lamellipodia may have a relatively longer lifetime and therefore a greater likelihood of becoming consolidated.

While newly extended distal growth cone areas appear to increase their substrate adhesion during consolidation, the proximal cone area, by contrast, appears to decrease its relatively tight apposition to the substrate during its transition to neuritic shaft. This reciprocal relationship between the adhesion properties of different growth cone regions during elongation may relate to recent evidence linking both serine proteases and protease inhibitors to the mechanism of neurite outgrowth (Krystosek and Seeds, 1981; Monard, 1985).

\section{Mechanistic considerations}

The speed and extent of the shape changes we observed in the growth cone suggest that posttranslational protein modifications are likely to be involved. For example, many rapid NGF-dependent changes in protein phosphorylation have been demonstrated (Halegoua and Patrick, 1980; Yu et al., 1980; Greene et al., 1983; Landreth and Rieser, 1985). Microfilaments (Fine and Bray, 1971) and microtubules represent 2 substrates of probable importance in the NGF-promoted elongation. Although the most distal regions of growth cones contain few, if any, microtubule bundles (Yamada et al., 1971; Bunge, 1973; Cheng and Reese, 1985), the latter are abundant in the neuritic shaft. Thus, the transition of growth cone to shaft during elongation must include the formation of microtubules. Recent findings suggest that this process occurs near the neurite tip (Bamburg et al., 1986). Also, NGF promotes the stability (Black and Greene, 1982) and assembly (Black et al., 1986) of tubulin in PC1 2 cells, and there is evidence that this may occur via NGFdependent phosphorylation of microtubule-associated proteins (Burstein et al., 1985; Black et al., 1986). Aside from providing structural support for the elongating neurite, microtubules are also involved in the important function of transporting material to the growing tip.

Future analyses of the dynamic cytoskeletal restructuring and of the protein modifications that are likely to occur during growth will obviously require biochemical approaches, but their power can be greatly enhanced and their interpretations functionally tested in conjunction with the video microscopy (AVEC-DIC) of living cells in culture used as a biological assay system.

\section{References}

Abercrombie, M., and E. J. Ambrose (1958) Interference microscope studies of cell contacts in tissue culture. Exp. Cell Res. 15: 332-345.

Abercrombie, M., J. E. M. Heaysman, and S. M. Pegrum (1970) The locomotion of fibroblasts in culture II. "Ruffling." Exp. Cell Res. 60: $437-444$.

Aletta, J. M., L. A. Greene, and J. C. Edmondson (1986) Cytological features observed during NGF-dependent neurite elongation and growth cone motility in PC12 cells. Soc. Neurosci. Abstr. 12: 368.

Allen, R. D., N. Stromgren Allen, and J. L. Travis (1981) Videoenhanced contrast, differential interference contrast (AVEC-DIC) microscopy: A new method capable of analyzing microtubule-related motility in the reticulopodial network of Allogromia latticollaris. Cell Motil. 1: 291-302. 
Argiro, V., M. B. Bunge, and M. I. Johnson (1984) Correlation between growth cone form and movement and their dependence on neuronal age. J. Neurosci. 4: 3051-3062.

Bailey, N. T. J. (1959) Statistical Methods in Biology, pp. 61-65, Wiley, New York.

Bamburg, J. R., D. Bray, and K. Chapman (1986) Assembly of microtubules at the tip of growing axons. Nature 321: 788-790.

Bentley, D., and A. Toroian-Raymond (1986) Disoriented pathfinding hy pioneer neurone growth cones deprived of filopodia by cytochalasin treatment. Nature 323: 712-715.

Black, M. M., and L. A. Greene (1982) Changes in the colchicine susceptibility of microtubules associated with neurite outgrowth: Studies with nerve growth factor-responsive $\mathrm{PC} 12$ pheochromocytoma cells. J. Cell Biol. 95: 379-386.

Black, M. M., J. M. Aletta, and L. A. Greene (1986) Regulation of microtubule composition and stability during nerve growth factorpromoted neurite outgrowth. J. Cell Biol. 103: 545-557.

Bornstein, M. B. (1958) Reconstituted rat-tail collagen used as a substrate for tissue cultures on coverslips in Maximow slides and roller tubes. Lab. Invest. 2: 134-137.

Bovolenta, P., and C. Mason (1987) Growth cone morphology varies with position in the developing mouse visual pathway from retina to first targets. J. Neurosci. 7: 1447-1460.

Bray, D. (1970) Surface movements during the growth of single explanted neurons. Proc. Natl. Acad. Sci. USA 65: 905-910.

Bray, D. (1973) Model for membrane movements in the neural growth cone. Nature 244: 93-96.

Bray, D. (1982) Filopodial contraction and growth cone guidance. In Cell Behaviour, R. Bellairs, A. Curtis, and G. Dunn, eds., pp. 299 317, Cambridge U. P., Cambridge, UK

Bray, D., and M. B. Bunge (1973) The growth cone in neurite extension, In Locomotion of Tissue Cells, R. Porter and D. W. Fitzsimons, eds., pp. 195-209, Elsevier, Amsterdam.

Bray, D., and K. Chapman (1985) Analysis of microspike movements on the neuronal growth cone. J. Neurosci. 5: 3204-3213.

Bunge, M. B. (1973) Fine structure of nerve fibers and growth cones of isolated sympathetic neurons in culture. J. Cell Biol. 56: 713-735.

Bunge, M. B., M. I. Johnson, and V. J. Argiro (1983) Studies of regenerating nerve fibers and growth cones. In Spinal Cord Reconstruction, C. C. Kao, R. P. Bunge, and P. J. Reier, eds., pp. 99-120, Raven, New York.

Burstein, D. E., P. J. Seeley, and L. A. Greene (1985) Lithium ion inhibits nerve growth factor-induced neurite outgrowth and phosphorylation of nerve growth factor-modulated microtubule-associated proteins. J. Cell Biol. 101: 862-870.

Caudy, M., and D. Bentley (1986) Pioneer growth cone steering along a series of different affinities. J. Neurosci. 6: 1781-1795.

Cheng, T. P. O., and T. S. Reese (1985) Polarized compartmentalization of organelles in growth cones from developing optic tectum. J. Cell Biol. 101: 1473-1480.

Connolly, J. L., P. J. Seeley, and L. A. Greene (1985) Regulation of growth cone morphology by nerve growth factor: A comparative study by scanning electron microscopy. J. Neurosci. Res. 13: 183-198.

Connolly, J. L., P. J, Seeley, and L. A. Greene (1987) Rapid regulation of neuronal growth cone shape and surface morphology by nerve growth factor. Neurochem. Res. 12: 861-868.

Edmondson, J. C., and M. E. Hatten (1987) Glial-guided granulc neuron migration in vitro: A high resolution time-lapse video microscopic study. J. Neurosci. 7: 1928-1934.

Fine, R. E., and D. Bray (1971) Actin in growing nerve cells. Nature 234: $115-118$.

Goldberg, D. J., and D. W. Burmeister (1986) Stages in axon formation: Observations of growth of Aplysia axons in culture using video-enhanced contrast-differential interference contrast microscopy. J. Cell Biol. 103: 1921-1931.

Gravel, C., N. Leclerc, A. Plioplys, and R. B. Hawkes (1986) Focal axonal swellings in rat cerebellar Purkinje cells during normal development. Brain Res. 363: 325-332.

Greene, L. A., and A. S. Tischler (1976) Establishment of a noradrenergic clonal line of rat adrenal pheochromocytoma cells which respond to ncrve growth factor. Proc. Natl. Acad. Sci. USA 73: 2424 2428.

Greene, L. A., R. K. H. Liem, and M. L. Shelanski (1983) Regulation of a high molecular weight microtubule-associated protein in $\mathrm{PCl} 2$ cells by nerve growth factor. J. Cell Biol. 96: 76-83.
Grillo, M. A. (1966) Electron microscopy of sympathetic tissues. Pharmacol. Rev. 18: 387-399.

Gundersen, R. W., and J. N. Barrett (1980) Characterization of the turning response of dorsal root neurites toward NGF. J. Cell Biol. 87: 546-554.

Halegoua, S., and J. Patrick (1980) Nerve growth factor mediates phosphorylation of specific proteins. Cell 22: 571-581.

Hammarback, J. A., and P. C. Letourneau (1986) Neurite extension across regions of low cell-substratum adhesivity: Implications for the guidepost hypothesis of axonal pathfinding. Dev. Biol. 117:655-662.

Haydon, P. G., C. S. Cohan, D. P. McCobb, H. R. Miller, and S. B. Kater (1985) Neuron-specific growth cone properties as seen in identified neurons of Helisoma. J: Neurosci. Res. 13: 135-147.

Horie, H., T. Takenaka, and M. Kaiho (1983) Effects of disruption of microtubules on translocation of particles and morphology in tissue cultured neurites. Brain Res. 288: 85-93.

Ingram, V. M. (1969) A side view of moving fibroblasts. Nature 222: 641-644.

Jacobs, J. R., and J. K. Stevens (1986) Experimental modification of PC12 neurite shape with the microtubule-depolymerizing drug nocodazole: A serial electron microscopic study of neurite shape control. J. Cell Biol. 103: 907-915.

Johnston, R. N., and N. K. Wessells (1980) Regulation of the elongating nerve fiber. Curr. Top. Dev. Biol. 16: 165-206.

Koenig, E., S. Kinsman, E. Repasky, and L. Sultz (1985) Rapid mobility of motile varicosities and inclusions containing a-spectrin, actin, and calmodulin in regenerating axons in vitro. J. Neurosci. 5: 715729.

Krystosek, A., and N. W. Seeds (1981) Plasminogen activator release at the neuronal growth cone. Science 213: 1532-1534.

Landreth, G. E., and G. E. Rieser (1985) Nerve growth factor- and epidermal growth factor-stimulated phosphorylation of a $\mathrm{PC1} 2 \mathrm{cy}-$ toskeletally associated protein in situ. J. Cell Biol. 100: 677-683.

Lasek, R. J. (1981) The dynamic ordering of neuronal cytoskeletons. Neurosci. Res. Prog. Bull. 19: 7-32.

Letourneau, P. C. (1979) Cell-substratum adhesion of neurite growth cones, and its role in neurite elongation. Exp. Cell Res. 124: 127138.

Marsh, L., and P. C. Letourneau (1984) Growth of neurites without filopodial or lamellipodial activity in the presence of cytochalasin B. J. Cell Biol. 99: 2041-2047.

Mobley, W. C., A. Schenker, and E. M. Shooter (1976) Characterization and isolation of proteolytically modified nerve growth factor. Biochemistry 15: 5543-5552.

Monard, D. (1985) Implications of proteases and protease inhibitors in neurite outgrowth. In Neurobiochemistry, B. Hamprecht and V. Neuhoff, eds., pp. 7-12, Springer-Verlag, Berlin.

Nakai, J., and Y. Kawasaki (1959) Studies on the mechanism determining the course of nerve fibers in tissue culture. I. The reaction of the cone to various obstructions. Z. Zellforsch. 51: 108-122.

Pfenninger, K. H., and M. Maylie-Pfenninger (1981) Lectin labeling of sprouting neurons II. Relative movement and appearance of glycoconjugates during plasmalemmal expansion. J. Cell Biol. 89: 547559.

Porter, R., and D. W. Fitzsimons (eds.) (1973) Locomotion of Tissue Cells, Elsevier, Amsterdam.

Sceley, P. J., and L. A. Greenc (1983) Short-latency local actions of nerve growth factor at the growth cone. Proc. Natl. Acad. Sci. USA 80: 2789-2793.

Tosney, K. W., and L. T. Landmesser (1985) Growth cone morphology and the trajectory in the lumbosacral region of the chick embryo. J. Neurosci. 5: 2345-2358.

Tosney, K. W., and N. K. Wessells (1983) Neuronal motility: The ultrastructure of veils and microspikes correlates with their motile activities. J. Cell. Sci. 61: 389-411.

Weiss, P., and H. B. Hiscoe (1948) Experiments on the mechanism of nerve growth. J. Exp. Zool. 107: 315-395.

Yamada, K. M., B. S. Spooner, and N. K. Wessells (1971) Ultrastructure and function of growth cones and axons of cultured nerve cells. J. Cell Biol. 49: 614-635.

Yu, M. W., N. W. Tolson, and G. Guroff (1980) Increased phosphorylation of specific nuclear proteins in superior cervical ganglia and PC1 2 cells in response to nerve growth factor. J. Biol. Chem. 255: $10481-10492$. 